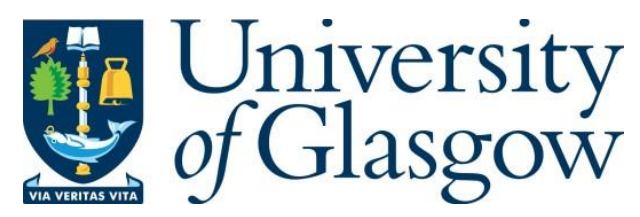

Perera, D. et al. (2018) Percutaneous revascularization for ischemic ventricular dysfunction: rationale and design of the REVIVED-BCIS2 trial: percutaneous coronary intervention for ischemic cardiomyopathy. JACC: Heart Failure, 6(6), pp. 517-526.

There may be differences between this version and the published version. You are advised to consult the publisher's version if you wish to cite from it.

http://eprints.gla.ac.uk/163793/

Deposited on: 02 August 2018

Enlighten - Research publications by members of the University of Glasgow http://eprints.gla.ac.uk 


\section{Percutaneous Revascularization for Ischemic Ventricular Dysfunction: rationale and design of the REVIVED-BCIS2 trial}

Divaka Perera ${ }^{1}$, Tim Clayton ${ }^{2}$, Mark C Petrie ${ }^{3}$, John P Greenwood ${ }^{4}$, Peter D O'Kane ${ }^{5}$, Richard Evans ${ }^{2}$, Mark Sculpher6, Theresa Mcdonagh ${ }^{7}$, Anthony Gershlick $^{8}$, Mark de Belder ${ }^{9}$, Simon Redwood ${ }^{1}$, Gerald Carr-White ${ }^{1}$, Michael Marber ${ }^{1}$ on behalf of the REVIVED investigators

1. National Institute for Health Research Biomedical Research Centre and British Heart Foundation Centre of Excellence, School of Cardiovascular Medicine and Sciences, King's College London, London, UK

2. Clinical Trials Unit, London School of Hygiene and Tropical Medicine, London, UK 3. Institute of Cardiovascular and Medical Sciences, University of Glasgow, UK

4. Leeds Institute of Cardiovascular and Metabolic Medicine, University of Leeds, UK

5. Royal Bournemouth and Christchurch Hospital, UK

6. Centre for Health Economics, University of York, UK.

7. King's College Hospital, London, UK

8. Biomedical Research Unit, University Hospitals of Leicester, Leicester, UK

9. The James Cook Hospital, Middlesbrough, UK

Correspondence to:

Prof. Divaka Perera, Cardiovascular Division, Rayne Institute, $4^{\text {th }}$ Floor Lambeth Wing, St Thomas' Hospital, London, SE1 7EH, UK

(Divaka.Perera@kcl.ac.uk, +4420 7188 1048)

Key Words: Ischemic Cardiomyopathy, Percutaneous Coronary Intervention, Left

Ventricular Dysfunction, Myocardial Viability, Heart Failure 


\section{Commonly used abbreviations}

BNP: $\quad$ Brain-type natriuretic peptide

BCIS-JS: British Cardiovascular Intervention Society jeopardy score

CABG: $\quad$ Coronary artery bypass graft surgery

EF: $\quad$ Ejection fraction

HF: $\quad$ Heart failure

ICD: Implantable cardioverter defibrillator

ICM: Ischemic cardiomyopathy

LV: $\quad$ Left ventricular

MRI: $\quad$ Magnetic resonance imaging

MI: $\quad$ Myocardial infarction

OMT: $\quad$ Optimal medical therapy

PCI: $\quad$ Percutaneous coronary intervention

\section{Condensed Abstract}

Heart failure (HF) due to coronary artery disease is associated with significant mortality and morbidity. REVIVED-BCIS2 is a prospective, multi-center, openlabel, randomized controlled trial that addresses the hypothesis that PCI in combination with optimal medical therapy (OMT) will reduce all-cause death and hospitalization for HF compared to a strategy of OMT alone in patients with a left ventricular ejection fraction $(\mathrm{LVEF}) \leq 35 \%$, extensive coronary disease and demonstrable myocardial viability. Change in LVEF is a major secondary endpoint. Follow-up will be for at least 2 years from randomization. 400 of 700 patients have been enrolled to date. 


\begin{abstract}
Background: Ischemic cardiomyopathy (ICM) is the commonest cause of heart failure (HF) and is associated with significant mortality and morbidity. Surgical revascularization has been shown to improve long-term outcomes in some patients, but surgery itself carries a major early hazard. Percutaneous coronary intervention (PCI) may allow a better balance between risk and benefit.
\end{abstract}

Objectives: Evaluate whether PCI in combination with optimal medical therapy (OMT) will reduce all-cause death and hospitalization for HF compared to a strategy of OMT alone.

Methods: REVIVED-BCIS2 is a prospective, multi-center, open- label, randomized controlled trial, funded by the National Institute for Health Research in the United Kingdom. Follow-up will be for at least 2 years from randomization. Secondary outcomes include left ventricular (LV) ejection fraction (EF), quality of life scores, appropriate Implantable Cardioverter Defibrillator therapy and acute myocardial infarction. Patients with LVEF $\leq 35 \%$, extensive coronary disease and demonstrable myocardial viability are eligible for inclusion and those with a myocardial infarction within 4 weeks, decompensated HF or sustained ventricular arrhythmias within 72 hours are excluded. A trial of 700 patients has more than $85 \%$ power to detect a $30 \%$ relative reduction in hazard.

Results: 400 patients have been enrolled to date.

Conclusion: International guidelines do not provide firm recommendations on the role of PCI in managing severe ICM, due to lack of robust evidence. REVIVEDBCIS2 will provide the first randomized data on the efficacy and safety of PCI in ICM and has the potential to inform guidelines pertaining to both revascularization and $\mathrm{HF}$. 


\section{Introduction}

The prevalence of heart failure (HF) due to left ventricular (LV) systolic dysfunction is increasing (1) and ischemic cardiomyopathy (ICM) accounts for approximately $60 \%$ of all HF cases(2,3). Pathophysiologically, ICM encompasses a spectrum of sequelae of coronary disease, including myocardial infarction (MI) (which leads to irreversible fibrosis) and hibernation (a potentially reversible adaptation to repetitive ischemia), which often co-exist in a given patient and can both lead to adverse remodeling and LV dysfunction. Hibernation was a term coined nearly 40 years ago to describe the reversal of remodeling and augmentation of systolic function following surgical coronary artery bypass grafting (CABG), noted in patients with chronic stable angina and severe LV dysfunction(4). While subsequent observational studies of surgical revascularization appeared to confirm the existence of hibernation(5, 6), until recently, this had not been adequately assessed in a randomized study.

The seminal Surgical Treatment for Ischemic Heart Failure (STICH) trial, the only randomized evaluation of CABG for ICM to date, enrolled patients with a LV ejection fraction $(E F) \leq 35 \%$. At a median of 4.6 years, the primary outcome, allcause mortality, was not significantly different between patients treated with optimal medical therapy (OMT) alone compared to those assigned to CABG surgery ( $41 \%$ vs. 36\%, hazard ratio (HR) 0.86, 95\% confidence interval (CI) 0.72 to $1.04, \mathrm{p}=0.12)(7)$. Mortality in the first 30 days was significantly higher in the surgical group (4\% vs. 1\%, HR 3.12, 95\% CI $1.33-7.32$, p=0.009). This finding is in keeping with the known association between mortality and LV dysfunction following CABG surgery(8). The early hazard of CABG may have negated the benefits of revascularization, which become gradually manifest in those who survive the complications of surgery. The Surgical Treatment for Ischemic Heart Failure Extension Study (STICHES) reported longer-term mortality data from the STICH trial. At median follow up of approximately 10 years, 59\% of patients assigned to CABG died versus 66\% in the medical therapy group (HR 0.84; $95 \%$ CI 0.73-0.97; p=0.02)(9). Death from cardiovascular causes and several prespecified composite secondary endpoints also occurred less often in the CABG group. The critical balance between safety and efficacy is also borne out when 
examining the impact of age on treatment effect in STICH. Long-term survival benefit was most apparent in the youngest patients enrolled in the trial (in whom the risks of peri-procedural mortality and morbidity are lowest) and this benefit diminished with increasing age(10).

Given the lower procedural risks associated with percutaneous coronary intervention (PCI), it has the potential to allow the benefits of revascularization to be realized with fewer complications than CABG surgery, but this assertion is yet to be tested in a randomized trial. Table 1 summarizes randomized and observational studies of revascularization versus medical therapy published in the past 15 years and includes the proportion of patients treated by PCI. It should be noted that the risk of longer-term complications, such as restenosis and late stent thrombosis, in this population who tend to have complex coronary disease and multiple comorbidities, is largely unknown. While numerous comparisons have been made between PCI and CABG in patients with symptomatic coronary disease, most of the large randomised trials excluded patients with impaired LV function. Less than $2 \%$ of all patients included in the largest randomised controlled trial comparing PCI with CABG, SYNTAX, had significant LV impairment $(\mathrm{EF}<30 \%)$ at baseline $(11)$. We reported outcomes of PCI in 301 patients with severe ICM (mean EF 24\%), showing 30-day, 6-month and 4 year mortality rates of $1.3 \%, 6 \%$ and $33 \%$, respectively $(12,13)$. These results appear to compare favourably with the surgical data, but as these are not matched cohorts, further comparison is not possible. On the other hand, the degree of LV impairment is a known determinant of adverse outcome even in patients undergoing PCI(14) and whether this modality of revascularization would offer incremental prognostic benefit, over and above contemporary HF medication and device therapy, is unclear. A recent meta-analysis of observational data suggests that CABG may offer superior outcomes compared to PCI, with either modality being preferable to medial therapy alone(15). The 2014 ESC guidelines for revascularisation make a class IIb recommendation (with a level of evidence $\mathrm{C}$ ) for PCI, in the presence of viable myocardium, where surgery is not indicated(16). REVIVED-BCIS2 is the first randomised comparison of 
percutaneous revascularisation (with OMT) versus OMT alone in patients with LV dysfunction and viable myocardium.

\section{Trial hypotheses and outcome measures}

The principle hypothesis of REVIVED_BCIS2 is that PCI in combination with OMT will improve event-free survival in patients with ICM and viable myocardium, compared to a strategy of OMT alone. The main secondary hypothesis is that PCI will improve LV systolic function in this cohort compared to OMT alone. The primary outcome is a composite endpoint of all-cause death or hospitalization due to HF, over the entire duration of the trial. Patients will be followed up for at least 2 years from randomization (expected range 2 to 8.5 years). The major secondary outcome is LVEF, assessed by echocardiography, 6 and 12 months from randomization. Other outcome measures include cardiovascular death, allcause death, hospitalization due to $\mathrm{HF}$, acute MI, appropriate Implantable Cardioverter Defibrillator (ICD) therapy, quality of life scores (Kansas City Cardiomyopathy Questionnaire and EuroQol EQ-5D-5L), New York Heart Association (NYHA) functional class, unplanned further revascularization, Canadian Cardiovascular Society (CCS) angina class, health resource use, serial Troponin T or I levels, serial Brain-type Natriuretic Peptide (BNP or NT-proBNP) levels and the incidence of major bleeding. Definitions of outcome measures are detailed in table 2 .

\section{Study Population}

Individuals with all of the following characteristics will be eligible for inclusion: severe $\mathrm{LV}$ dysfunction ( $\mathrm{EF} \leq 35 \%$ ), extensive coronary disease and demonstrable viability in at least 4 dysfunctional myocardial segments(17) that can be revascularized by PCI. As this is a trial assessing the prognosis of patients with LV dysfunction, those with a spectrum of HF symptoms (NHYA I to IV) will be enrolled.

LVEF is assessed by the biplane Simpson's Rule/3D echocardiography or by Magnetic Resonance Imaging (MRI). If there has been a recent clinical diagnosis of MI (MI), the imaging study is performed at least 4 weeks after the MI. 
Extensive coronary disease is defined as a British Cardiovascular Intervention Society myocardial jeopardy score (BCIS-JS)(18) of at least 6 (the maximum possible score is 12; a calculation tool is included in the supplementary appendix). The BCIS-JS can be applied to patients with or without previous bypass grafts; for illustration, patients who do not have bypass grafts will have a BCIS-JS $\geq 6$ if they have significant left main, proximal LAD or at least proximal two-vessel disease. Myocardial viability is characterized using the AHA 17segment model and can be assessed using any recognised modality, including MRI, Dobutamine Stress Echocardiography (DSE), Single Photon Emission Computerised Tomography (SPECT) or Positron Emission Tomography (PET).

Trial exclusion criteria are a MI within 4 weeks of randomization (this is a clinical definition as adjudicated by recruiting centres); acutely decompensated HF requiring treatment with inotropes/ ventilation/MCS within 72 hours of randomization; sustained ventricular tachycardia/fibrillation (VT/VF) or appropriate ICD discharges within 72 hours of randomisation; valve disease deemed by the local heart team to require imminent intervention; any contraindications to PCI; age $<18$ yrs (there is no upper age limit); estimated glomerular filtration rate $<25 \mathrm{ml} / \mathrm{min} / 1.73 \mathrm{~m}^{2}$, unless established on dialysis; pregnancy; previous enrolment in REVIVED-BCIS2 or current enrolment in other trial that may affect REVIVED-BCIS2 outcome data and life expectancy $<1$ year due to non-cardiac pathology.

It is anticipated that some eligible candidates (such as those with severe limiting angina) will be considered for revascularization on clinical grounds, at the discretion of the responsible clinician and in accordance with the wishes of the patients. Similarly, in some cases, eligible patients may be offered coronary artery bypass surgery, including those thought to benefit from adjunctive surgical procedures (like valve repair/replacement or left ventricular reconstruction) or those whose coronary anatomy is considered by the local team to be more amenable to surgical rather than percutaneous revascularization. These patients will not be enrolled in the trial but the screening log (see below) will capture such exclusions. 


\section{Trial design, conduct and organization}

REVIVED-BCIS2 is a prospective randomized controlled trial, conducted across 30-35 centers in the United Kingdom. Once the principal investigator at each site confirms the eligibility of a patient and written informed consent is obtained, randomization is carried out via an online web-based system. Randomization of the treatment assignment is stratified by center using randomly permuted blocks of varying size, with 1:1 allocation between the PCI and OMT arms. Given the nature of PCI, this is an open-label trial, but researchers adjudicating and analysing trial outcomes will be blinded to treatment assignment. Figure 1 summarizes recruitment and study flow.

The trial is sponsored by King's College London, UK and funded by the UK Department of Health via the National Institute for Health Research (NIHR) (Health Technology Assessment project 10/57/67) with oversight by a Trial Steering Committee (TSC) that meets pre-specified independence criteria (Figure 3). A Data and Safety Monitoring Committee (DSMC) has been convened and a DSMC charter developed, which includes details of the meeting schedule and stopping guidelines. The DSMC are independent of the trial team and report directly to the TSC. The Clinical Trials Unit (CTU) at the London School of Hygiene and Tropical Medicine coordinate and monitor all aspects of the trial. The trial is officially endorsed by the British Cardiovascular Intervention Society (BCIS) and hence is referred to as REVIVED-BCIS2.

The protocol and amendments have been reviewed and approved by the UK National Research Ethics Service (London - Westminster committee; REC reference 10/H0802/46). The trial is carried out in accordance with the declaration of Helsinki and in keeping with Good Clinical Practice Guidelines. Registration was completed before recruitment commenced (NCT01920048) and ISRCTN45979711). The first patient was randomized on 28 th August 2013 and at the time of this publication, 400 patients have been randomized. Figure 2 summarizes the study timeline. There has been one major amendment to the protocol, implemented in July 2014, when the first inclusion criterion was 
modified from "LVEF $\leq 30 \%$ " to "LVEF $\leq 35 \%$ " to facilitate comparison with relevant literature and guidelines. At this stage, " $\geq$ CCS class 3 angina” was removed from the list of exclusion criteria, due to the difficulty in distinguishing angina from breathlessness in this particular population.

\section{Assessment of LV function and viability}

Suitability of patients on the basis of EF will be adjudicated by the participating centers, on the basis of recent echocardiography or MRI studies. All patients will also have echocardiography performed at randomization (if the qualifying EF was based on a recent echocardiogram, this can be submitted as the baseline study) as well as 6 and 12 months later. Baseline, 6 month and 12 month echocardiograms will be anonymized and submitted to an independent echocardiography core laboratory (at Guy's and St Thomas' Hospital, London, UK), which will determine LV volumes and EF using a biplane Simpson's method, for evaluation of the major secondary outcome. The core laboratory will be blinded to treatment assignment as well as to the timing of the studies in relation to randomization. Core laboratory analysis will also include the degree of mitral regurgitation and segmental wall motion.

Myocardial viability testing is used to prospectively predict hibernation by identifying the extent of fibrosis, contractile reserve, membrane integrity or metabolic activity(19). There has never been a randomized evaluation of the value of viability testing in the management of ICM and observational series have reported seemingly conflicting results. A meta-analysis of over 3000 patients with ICM from 24 studies showed that mortality was lower following revascularization in patients with viable myocardium but that this benefit was not seen in the absence of viability(20). A more recent observational series of patients with ICM assessed by PET showed that revascularization was associated with lower mortality compared to OMT when the extent of viability exceeded more than $10 \%$ of the whole myocardium(21). However, analysis of a subgroup of patients in the STICH trial who underwent discretionary viability testing, did not demonstrate an interaction between the response to revascularization and their viability classification(22). A pertinent consideration is the fact that the 
STICH substudy classified patients dichotomously as having viable hearts or not. However, an individual with ICM usually has some regions that are clearly viable and others that are not and with PCI, it is possible to target revascularization to myocardial territories selected on this basis. Notwithstanding differences in sensitivity and specificity between imaging modalities, in order to ensure widespread applicability of trial results, segmental viability will be determined by any recognized modality in REVIVED. Imaging and intervention specialists at each participating center assess segmental viability and the feasibility of revascularizing the relevant segments, to determine whether an individual patient will be eligible for randomization.

\section{Percutaneous coronary intervention arm}

PCI will be performed according to local protocols. Dual antiplatelet therapy should be given in all cases, with pre-loading, and the post-PCI duration based on the individual's bleeding risk and local/national guidelines. In general, drugeluting stents are recommended, but in patients who have an indication for longterm formal anticoagulation (e.g. for concurrent atrial fibrillation, LV thrombus or venous thromboembolic disease), the choice of stent type should be based on their suitability for medium-term combined antiplatelet and anticoagulation therapy.

Completeness of revascularization: it is strongly recommended that PCI be attempted on all significant coronary lesions in major proximal coronary vessels (or side branches $>2.5 \mathrm{~mm}$ in diameter) subtending viable myocardium. Lesion significance is defined as $>70 \%$ diameter stenosis on angiography or for lesions between 50 and $70 \%$ diameter stenosis, when accompanied by demonstrable reversible ischemia on invasive or non-invasive testing. Planned target lesions will need to be identified by the operator and recorded by the trial coordinator before the procedure. Patients who meet inclusion criteria and have chronic total occlusion (CTO) of coronary arteries subtending viable myocardial segments should be considered for REVIVED, provided that the PCI operators predict a high likelihood of successfully reopening these vessels. It is recommended that dedicated CTO operators, in units that have this degree of 
specialization, undertake such cases. The coronary disease burden at baseline and the completeness of final revascularization will be characterized by the BCIS-JS and Revascularization Index (RI), where RI = (JS pre $\left.-J S_{\text {post }}\right) / J S_{\text {pre }}(18)$. The interaction between treatment effect and RI as well as the presence of a CTO will be the subject of a separate substudy.

Staged PCI: a single stage strategy should be employed where possible. However, provisional staging could be considered in patients with renal dysfunction, complex coronary disease (including CTO) or if it is felt during PCI that deferring intervention to one or more vessels is in the patient's best interests (e.g. due to unexpected high contrast volumes or procedural complications during PCI to the first vessel). Staging must be prespecified at the index procedure. Urgent revascularization before the planned second stage procedure will be considered a secondary endpoint.

\section{Optimal medical therapy in both arms}

In order to ensure that patients in both arms of the trial receive optimal medical and device therapy, there is a nominated heart failure lead at each participating centre who is actively involved in patient selection and monitoring of therapy during the course of the trial. Furthermore, a trial Medical Therapy Committee has been established, that will review available evidence and guidelines at least annually and refine recommendations to ensure that drug and device therapy given to all patients in the trial remains optimal and contemporary. Each site is provided with a standard operating procedure for delivering and monitoring OMT, which sets out classes of drugs appropriate for trial patients, including HF therapies (such as angiotensin converting enzyme inhibitor or angiotensin receptor blocker +/- neprilysin inhibitor, betablocker and mineralocorticoid receptor antagonist (23)) and secondary prevention for atherosclerosis (including statin and antiplatelet agent) as well as recommended treatment targets (including lipid profile, $\mathrm{HbA1c}$, resting heart rate). Formal anticoagulation for LV thrombus detected on imaging or as prophylaxis for severe LV dysfunction/ dyskinesis is at the discretion of the treating physician. 
Initiation of the above treatments, dose-titration and relevant monitoring is per local HF protocols.

Eligible patients are initiated on medical therapy prior to randomization and, in patients presenting with de novo HF, assessment of LV EF is deferred if they are not on appropriate medical therapy at presentation. Optimization of medical and device therapy will continue in both groups even after randomization, throughout the course of the trial.

ICD implantation is not mandatory for inclusion in REVIVED, although many patients who fulfill trial eligibility criteria may also be candidates for primary prevention ICDs. Participating sites are encouraged to follow international guidelines(23) when deciding on ICD or resynchronization device therapy and to make and document the decision to implant (or not implant) a device, before randomization.

\section{Statistical considerations}

Power Calculation: In the STICH trial, the rate of all cause death or hospitalization for $\mathrm{HF}$ at 5 years was $54 \%$ in the medical therapy group, with approximately $50 \%$ of events occurring in the first year and a steady rate thereafter(7). These data are similar to the 1 year rates of death or HF hospitalization reported in registries of Western European populations(24). On this basis, the predicted occurrence of death or hospitalization for HF at two years is $36 \%$ in the OMT group. The primary outcome will be measured over the entire trial duration, with a minimum follow-up duration of two years. A trial of 700 (350 in each group), with 300 patients experiencing a primary outcome, would have over $85 \%$ power to detect a hazard ratio of 0.7 (a $30 \%$ relative reduction in the hazard) at 5\% significance, allowing for up to $5 \%$ losses by the end of follow-up. The hazard ratio of 0.7 is considered clinically meaningful and in line with the magnitude of benefit observed across other treatment modalities in this population. For the major secondary endpoint, even half this sample size will provide $90 \%$ power to detect a minimum difference in $\mathrm{EF}$ of $4 \%$, assuming a standard deviation of $11 \%$. 
This trial will be a comparison of initial strategy, rather than technique; the projected event rates and hazard ratio allow for the fact that OMT patients may undergo subsequent revascularization. As such, no additional adjustments have been made to the power calculation to account for unplanned revascularization in the OMT arm. In patients assigned to receive OMT, revascularization by PCI or CABG during the trial would only be recommended in one of the following circumstances: readmission with an acute coronary syndrome (diagnosed on the basis of typical ischemic symptoms as well as a rise in cardiac biomarker levels or dynamic ST-segment deviation on ECG), deterioration in exertional angina to $\geq$ CCS class 3 symptoms or the occurrence of resistant ventricular arrhythmias considered to be ischemic in etiology.

Statistical Analysis: A detailed statistical analysis plan will be finalized before any data are analyzed by treatment assignment. Analysis of outcomes will be by treatment assignment, on an intention-to-treat basis. An unadjusted time-toevent analysis will be performed on the primary outcome using data across all follow-up, with time to the first event (or censoring) times measured from randomization. Hazard ratios together with associated confidence intervals will be calculated from the Cox proportional hazards model. Cumulative event rates will be calculated and presented using Kaplan-Meier time-to-event curves. As a measure of absolute treatment difference, cumulative event rates will be compared at 2 years. Each individual component of the primary composite outcome as well as other secondary time to event outcomes will be analyzed using the above methods. Losses to follow-up are expected to be minimal and patients will be included up until the time they experience the event or are censored. Any categorical outcome measures compared at specific time points will be examined using risk ratios and risk differences, confidence intervals and significance tests. Continuous variables will be analyzed and presented as mean treatment differences, confidence intervals and p-values derived from analysis of co-variance models or unpaired t-tests as appropriate (with appropriate transformation if necessary). 
Interim analyses by treatment assignment are not planned. A limited number of subgroups analyses will be performed, which will be detailed in the analysis plan. A risk model will be developed, based on interactions between variables and treatment in the Cox model, and used to examine whether the impact of treatment depends on a person's underlying risk.

\section{Health Economic Analysis}

The Centre for Health Economics at the University of York, UK will perform a formal health economic analysis. Data will be collected on health service resource use including length of inpatient stays, outpatient visits, use of primary care resources, use of cardiovascular medication and devices and subsequent cardiovascular procedures. Resource use will be valued in monetary terms using routine unit cost data relevant to the UK National Health Service (NHS). These will include NHS Reference Costs, British National Formulary drug prices and the Personal Social Services Research Unit (PSSRU) survey of unit costs.

A formal cost effectiveness of PCI in this population will be undertaken using a decision analytic framework, which will be a cohort model with states representing death and different levels of HF symptoms. Key features will include the quantification of health benefits in terms of quality-adjusted life years (QALYs) and the use of an NHS cost perspective. Standard decision rules will be used to assess cost effectiveness and extensive sensitivity analysis will be undertaken (probabilistic and deterministic) to assess the implications of uncertainty in the available evidence for cost-effectiveness. Heterogeneity in cost effectiveness between different sub-groups of patients will be assessed using methods consistent with those applied to clinical outcomes.

\section{Data Collection and Monitoring}

Each patient's demographic details, medical history, electrocardiogram, routine blood results, cardiac medication, LVEF, viability assessment, ICD interrogation result (if applicable) and the BCIS-JS are recorded at baseline. LVEF will be reassessed at 6 and 12 months as detailed above. ICD interrogation, quality of life scores, BNP (or NT-Pro BNP) level, Troponin (T or I) level and cardiac medication are recorded at 6,12 and 24 months post-randomization. All major 
outcomes and Serious Adverse Events are collected at 6,12 and 24 months for all patients and yearly thereafter for patients who have been randomized more than 2 years before the end of the trial. Additionally, patients who undergo revascularization (by treatment assignment or as an unplanned procedure) have Troponin levels checked before and after the procedure. Hospitalization and mortality will be tracked using national databases to ensure that any unreported major outcome events are identified. The DMSC will review serious adverse events and any other trial safety issues. The Clinical Trial Unit collects a snapshot of screening, from each center, twice a year. Recruiting centers capture details of all patients with extensive $\mathrm{CAD}$ and $\mathrm{EF} \leq 35 \%$ during this representative period. These data will be used to generate a Consort-style flowchart, describing the total population screened as well as the frequency and causes of patients excluded from the trial.

\section{Conclusion}

Ischemic cardiomyopathy is the commonest cause of HF and is associated with significant mortality and morbidity. Surgical revascularization has recently been shown to improve long-term outcomes in some patients, but surgery itself carries a major early hazard in this group. PCI is an appealing alternative to surgery, which may allow a better balance between risk and benefit, but this assertion has never been formally tested. REVIVED is the first randomized controlled trial of PCI for severe ischemic LV dysfunction and will provide important data that will inform guidelines on revascularization in ICM. 


\section{References}

1. Benjamin EJ, Blaha MJ, Chiuve SE, et al. Heart Disease and Stroke Statistics2017 Update: A Report From the American Heart Association. Circulation 2017;135:e146-e603.

2. Gheorghiade M, Sopko G, De Luca L, et al. Navigating the crossroads of coronary artery disease and heart failure. Circulation 2006;114:1202-1213.

3. McMurray JJV, Packer M, Desai AS, et al. Angiotensin-neprilysin inhibition versus enalapril in heart failure. N. Engl. J. Med. 2014;371:993-1004.

4. Rahimtoola SH. Coronary bypass surgery for chronic angina--1981. A perspective. Circulation 1982;65:225-241.

5. Alderman EL, Fisher LD, Litwin P, et al. Results of coronary artery surgery in patients with poor left ventricular function (CASS). Circulation 1983;68:785795.

6. O'Connor CM, Velazquez EJ, Gardner LH, et al. Comparison of coronary artery bypass grafting versus medical therapy on long-term outcome in patients with ischemic cardiomyopathy (a 25-year experience from the Duke Cardiovascular Disease Databank). Am. J. Cardiol. 2002;90:101-107.

7. Velazquez EJ, Lee KL, Deja MA, et al. Coronary-artery bypass surgery in patients with left ventricular dysfunction. N. Engl. J. Med. 2011;364:1607-1616.

8. Ståhle E, Bergström R, Edlund B, et al. Influence of left ventricular function on survival after coronary artery bypass grafting. Ann. Thorac. Surg. 1997;64:437444.

9. Velazquez EJ, Lee KL, Jones RH, et al. Coronary-Artery Bypass Surgery in Patients with Ischemic Cardiomyopathy. N. Engl. J. Med. 2016;374:1511-1520.

10. Petrie MC, Jhund PS, She L, et al. Ten-Year Outcomes After Coronary Artery Bypass Grafting According to Age in Patients With Heart Failure and Left Ventricular Systolic Dysfunction: An Analysis of the Extended Follow-Up of the STICH Trial (Surgical Treatment for Ischemic Heart Failure). Circulation 2016;134:1314-1324.

11. Serruys PW, Morice M-C, Kappetein AP, et al. Percutaneous coronary intervention versus coronary-artery bypass grafting for severe coronary artery disease. N. Engl. J. Med. 2009;360:961-972.

12. Perera D, Stables R, Thomas M, et al. Elective intra-aortic balloon counterpulsation during high-risk percutaneous coronary intervention: a randomized controlled trial. JAMA 2010;304:867-874.

13. Perera D, Stables R, Clayton T, et al. Long-term mortality data from the balloon pump-assisted coronary intervention study (BCIS-1): a randomized, controlled trial of elective balloon counterpulsation during high-risk 
percutaneous coronary intervention. Circulation 2013;127:207-212.

14. Mamas MA, Anderson SG, O'Kane PD, et al. Impact of left ventricular function in relation to procedural outcomes following percutaneous coronary intervention: insights from the British Cardiovascular Intervention Society. Eur. Heart J. 2014;35:3004-12a.

15. Wolff G, Dimitroulis D, Andreotti F, et al. Survival Benefits of Invasive Versus Conservative Strategies in Heart Failure in Patients With Reduced Ejection Fraction and Coronary Artery Disease: A Meta-Analysis. Circ Heart Fail 2017;10:e003255.

16. Authors/Task Force Members, Windecker S, Kolh P, et al. 2014 ESC/EACTS Guidelines on myocardial revascularization: The Task Force on Myocardial Revascularization of the European Society of Cardiology (ESC) and the European Association for Cardio-Thoracic Surgery (EACTS)Developed with the special contribution of the European Association of Percutaneous Cardiovascular Interventions (EAPCI). Eur. Heart J. 2014;35:2541-2619.

17. Cerqueira MD, Weissman NJ, Dilsizian V, et al. Standardized myocardial segmentation and nomenclature for tomographic imaging of the heart. A statement for healthcare professionals from the Cardiac Imaging Committee of the Council on Clinical Cardiology of the American Heart Association. Circulation. 2002 Jan 29;105(4):539-42.

18. De Silva K, Morton G, Sicard P, et al. Prognostic utility of BCIS myocardial jeopardy score for classification of coronary disease burden and completeness of revascularization. Am. J. Cardiol. 2013;111:172-177.

19. Schuster A, Morton G, Chiribiri A, Perera D, Vanoverschelde J-L, Nagel E. Imaging in the Management of Ischemic Cardiomyopathy. J. Am. Coll. Cardiol. 2012;59:359-370.

20. Allman KC, Shaw LJ, Hachamovitch R, Udelson JE. Myocardial viability testing and impact of revascularization on prognosis in patients with coronary artery disease and left ventricular dysfunction: a meta-analysis. J. Am. Coll. Cardiol. 2002;39:1151-1158.

21. Ling LF, Marwick TH, Flores DR, et al. Identification of therapeutic benefit from revascularization in patients with left ventricular systolic dysfunction: inducible ischemia versus hibernating myocardium. Circ Cardiovasc Imaging 2013;6:363-372.

22. Bonow RO, Maurer G, Lee KL, et al. Myocardial viability and survival in ischemic left ventricular dysfunction. N. Engl. J. Med. 2011;364:1617-1625.

23. Ponikowski P, Voors AA, Anker SD, et al. 2016 ESC Guidelines for the diagnosis and treatment of acute and chronic heart failure: The Task Force for the diagnosis and treatment of acute and chronic heart failure of the European Society of Cardiology (ESC)Developed with the special contribution of the Heart Failure Association (HFA) of the ESC. Eur. Heart J. 2016;37:2129-2200. 
24. Maggioni AP, Dahlström U, Filippatos G, et al. EURObservational Research Programme: regional differences and 1-year follow-up results of the Heart Failure Pilot Survey (ESC-HF Pilot). Eur. J. Heart Fail. 2013;15:808-817.

25. Gerber BL, Rousseau MF, Ahn SA, et al. Prognostic value of myocardial viability by delayed-enhanced magnetic resonance in patients with coronary artery disease and low ejection fraction: impact of revascularization therapy. J. Am. Coll. Cardiol. 2012;59:825-835.

26. AlJaroudi W, Alraies MC, Hachamovitch R, et al. Association of left ventricular mechanical dyssynchrony with survival benefit from revascularization: a study of gated positron emission tomography in patients with ischemic LV dysfunction and narrow QRS. Eur. J. Nucl. Med. Mol. Imaging 2012;39:1581-1591.

27. Velazquez EJ, Williams JB, Yow E, et al. Long-term survival of patients with ischemic cardiomyopathy treated by coronary artery bypass grafting versus medical therapy. Ann. Thorac. Surg. 2012;93:523-530.

28. Cleland JGF, Calvert M, Freemantle N, et al. The Heart Failure Revascularisation Trial (HEART). Eur. J. Heart Fail. 2011;13:227-233.

29. Sawada SG, Dasgupta S, Nguyen J, et al. Effect of revascularization on longterm survival in patients with ischemic left ventricular dysfunction and a wide range of viability. Am. J. Cardiol. 2010;106:187-192.

30. Desideri A, Cortigiani L, Christen AI, et al. The extent of perfusion-F18fluorodeoxyglucose positron emission tomography mismatch determines mortality in medically treated patients with chronic ischemic left ventricular dysfunction. J. Am. Coll. Cardiol. 2005;46:1264-1269.

31. Liao L, Cabell CH, Jollis JG, et al. Usefulness of myocardial viability or ischemia in predicting long-term survival for patients with severe left ventricular dysfunction undergoing revascularization. Am. J. Cardiol. 2004;93:1275-1279.

32. Meluzín J, Cerný J, Spinarová L, et al. Prognosis of patients with chronic coronary artery disease and severe left ventricular dysfunction. The importance of myocardial viability. Eur. J. Heart Fail. 2003;5:85-93.

33. Sicari R, Picano E, Cortigiani L, et al. Prognostic value of myocardial viability recognized by low-dose dobutamine echocardiography in chronic ischemic left ventricular dysfunction. Am. J. Cardiol. 2003;92:1263-1266.

34. Sawada SG, Lewis SJ, Foltz J, et al. Usefulness of rest and low-dose dobutamine wall motion scores in predicting survival and benefit from revascularization in patients with ischemic cardiomyopathy. Am. J. Cardiol. 2002;89:811-816.

35. Shah BR, Velazquez E, Shaw LK, Bart B, O'Connor C, Wagner GS. Revascularization improves survival in ischemic cardiomyopathy regardless of electrocardiographic criteria for prior small-to-medium myocardial infarcts. Am. Heart J. 2002;143:111-117. 
36. Thygesen K, Joint ESC/ACCF/AHA/WHF Task Force for Universal Definition of Myocardial Infarction, Authors/Task Force Members Chairpersons, et al. Third universal definition of myocardial infarction. J Am Coll Cardiol.

2012;60(16):1581-98.

37. Hicks KA, Tcheng JE, Bozkurt B, et al. 2014 ACC/AHA Key Data Elements and Definitions for Cardiovascular Endpoint Events in Clinical Trials: A Report of the American College of Cardiology/American Heart Association Task Force on Clinical Data Standards (Writing Committee to Develop Cardiovascular Endpoints Data Standards). J. Am. Coll. Cardiol. 2015;66:403-469.

38. Mehran R, Rao SV, Bhatt DL, et al. Standardized bleeding definitions for cardiovascular clinical trials: a consensus report from the Bleeding Academic Research Consortium. J Am Heart Assoc 2011;123:2736-47. 
Figure 1: STUDY FLOW

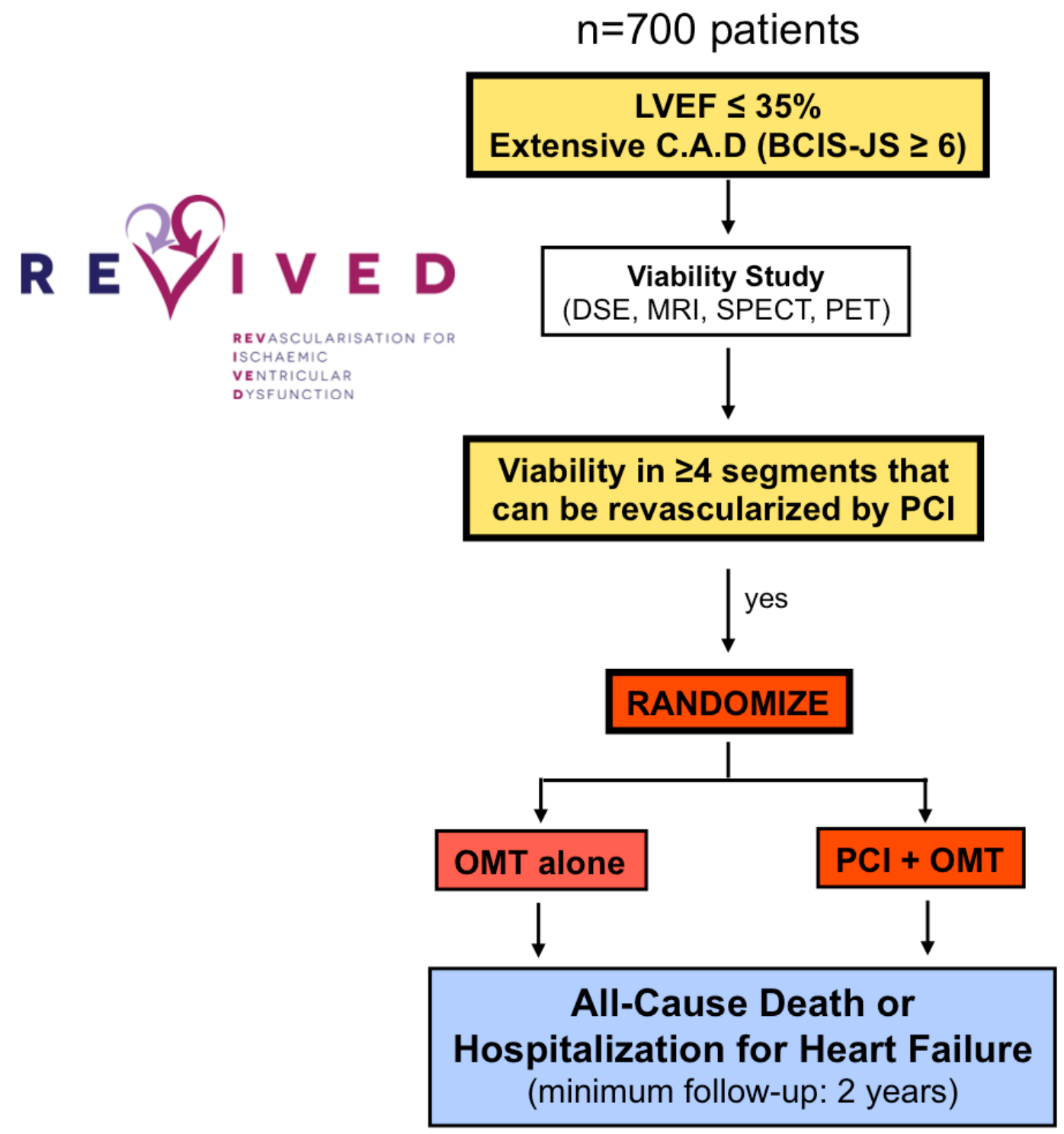


Figure 2: STUDY TIMELINE

No. of

patients

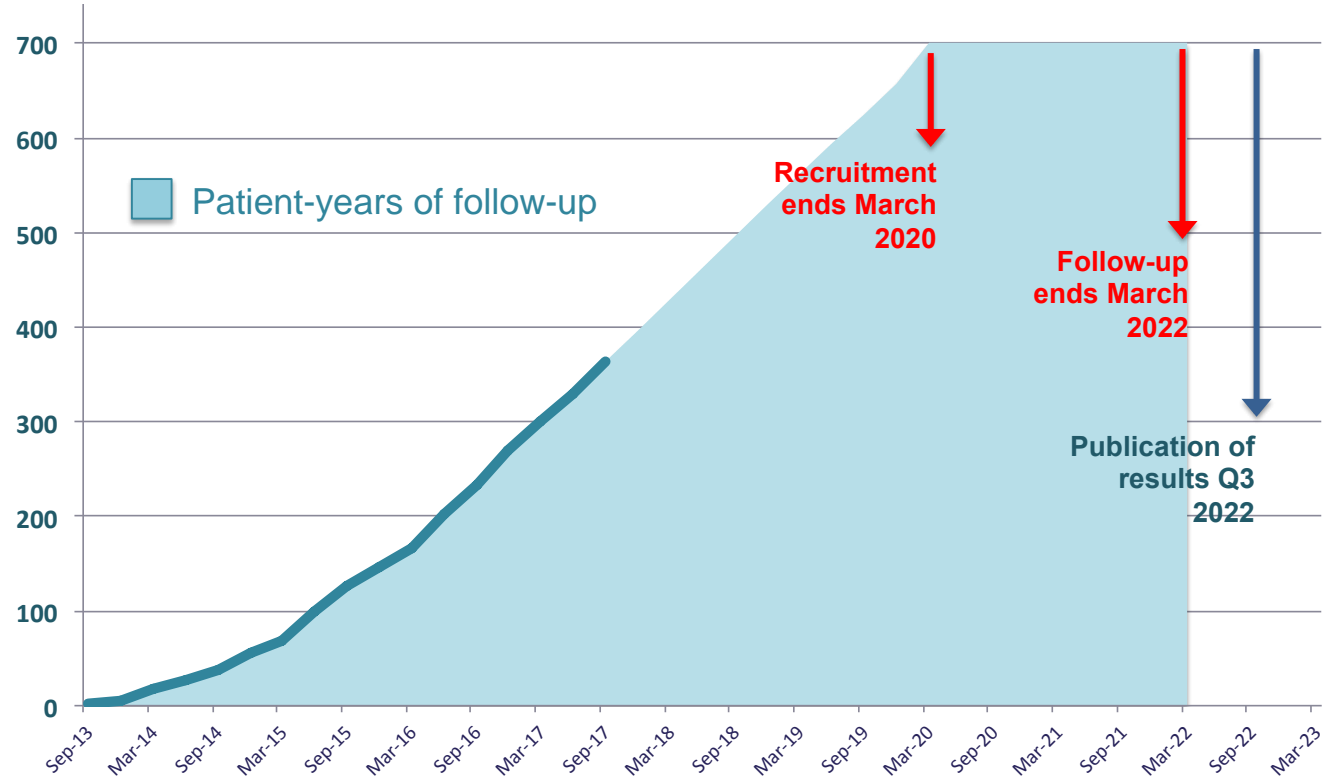

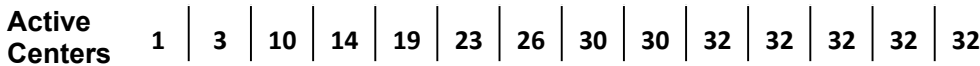


Figure 3: Trial Organization

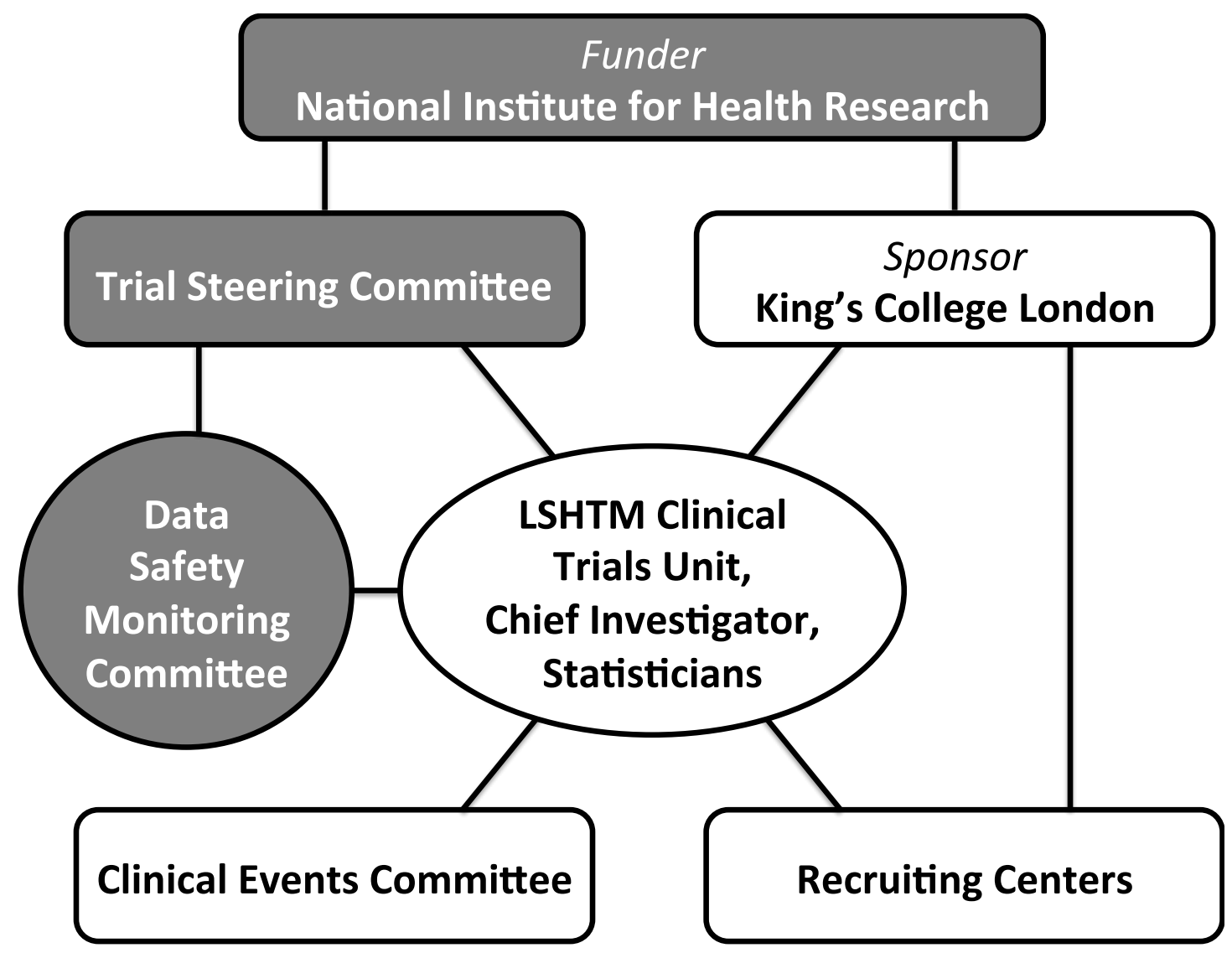


Table 1. Studies of revascularization versus medical therapy published between 2002 and 2017

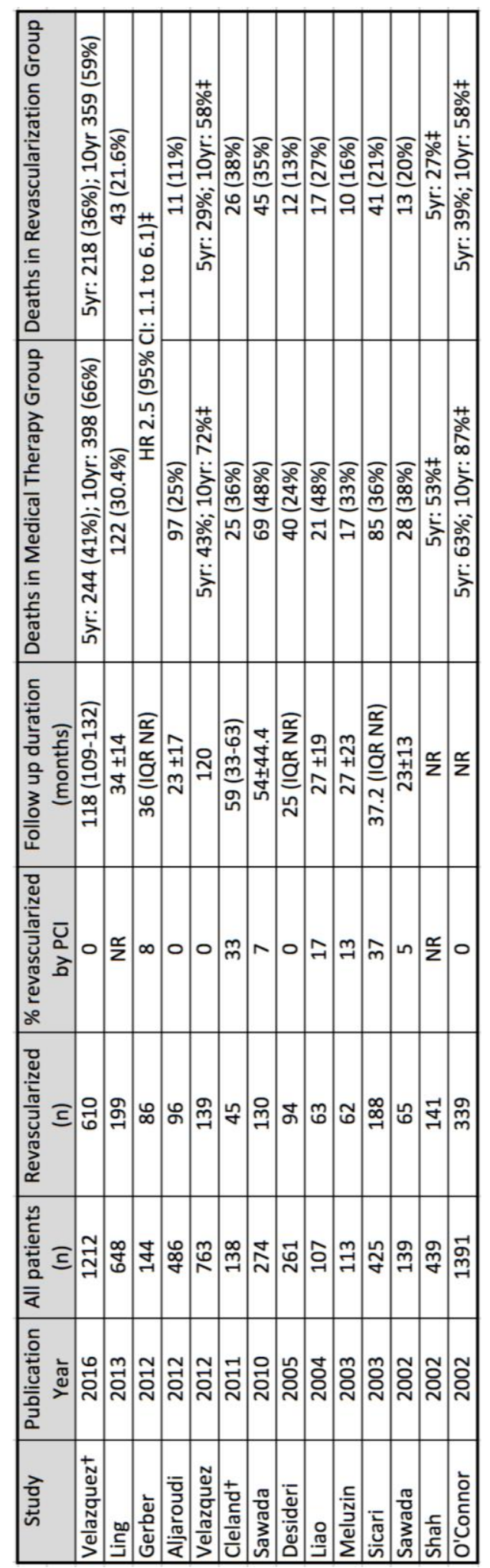

Follow-up duration is quoted as mean \pm SD or median (IQR).

$\dagger$ RCTs, $\neq$ adjusted mortality/propensity matched data; NR: not reported $(6,7,9,21,25-35)$ 


\section{Table 2. Definitions of outcome measures}

\begin{tabular}{|c|c|}
\hline $\begin{array}{l}\text { Acute Myocardial } \\
\text { Infarction (MI) }\end{array}$ & $\begin{array}{l}\text { 1. Spontaneous MI ( } \geq 48 \text { hrs after PCI/CABG) } \\
\text { Detection of a rise and/or fall of cardiac Troponin T or I levels, } \\
\text { with at least one value higher than the 99th percentile upper } \\
\text { reference limit (URL) AND symptoms consistent with } \\
\text { ischaemia OR dynamic ECG changes (including }>1 \mathrm{~mm} \text { ST } \\
\text { elevation, new Left Bundle Branch Block (LBBB) }>1 \mathrm{~mm} \text { ST } \\
\text { depression, }>3 \mathrm{~mm} \text { T wave inversion) } \\
\text { 2. Peri-procedural MI (<48 hrs after PCI/CABG)* } \\
\text { Following PCI, Troponin (T or I) > } 5 \text { x the 99th percentile } \\
\text { URL) (or } 5 \text { x the baseline value if this is higher than the URL) } \\
\text { in combination with any of (a) evidence of prolonged } \\
\text { ischaemia (>20 min) as demonstrated by prolonged chest pain } \\
\text { and/or ischaemic ST changes or (b) new pathological Q waves } \\
\text { or (c) angiographic evidence of a flow limiting complication, } \\
\text { such as of loss of patency of a side branch, persistent slow- } \\
\text { flow or no-reflow, embolisation, or (d) imaging evidence of } \\
\text { new loss of viable myocardium or new regional wall motion } \\
\text { abnormality. } \\
\text { Following CABG, Troponin (T or I) > } 10 \text { x 99th percentile URL } \\
\text { (or } 10 \mathrm{x} \text { the baseline value if this is higher than the URL) in } \\
\text { combination with any of the following: (i) new pathological Q } \\
\text { waves or (ii) angiographically documented new graft or new } \\
\text { native coronary artery occlusion or (iii) imaging evidence of } \\
\text { new loss of viable myocardium or new regional wall motion } \\
\text { abnormality, } \\
\text { 3. Sudden death } \\
\text { Cardiac arrest accompanied by new ST elevation/LBBB on } \\
\text { ECG and/or evidence of fresh coronary thrombus at } \\
\text { autopsy/angiography } \\
\text { a periprocedural MI or not on the basis of the } 2012 \text { Universal } \\
\text { Definition of a type } 4 \text { MI( } 36 \text { ), baseline and peak Troponin levels } \\
\text { measured within } 24 \text { hours of a procedure will be recorded. This will } \\
\text { provide a continuous outcome measure of periprocedural myocardial } \\
\text { injury and will also allow subsequent reclassification in the event of } \\
\text { further revisions to definitions of periprocedural MI that may occur } \\
\text { during the course of the trial.. }\end{array}$ \\
\hline $\begin{array}{l}\text { Appropriate ICD } \\
\text { therapy }\end{array}$ & $\begin{array}{l}\text { At least one ICD shock or episode of anti-tachycardia pacing } \\
\text { for documented ventricular tachycardia (VT) or ventricular } \\
\text { fibrillation (VF) }\end{array}$ \\
\hline
\end{tabular}




\begin{tabular}{|c|c|}
\hline $\begin{array}{l}\text { Cardiovascular } \\
\text { death }\end{array}$ & $\begin{array}{l}\text { All deaths where there is no clinical or post-mortem evidence } \\
\text { of a non cardiovascular aetiology }\end{array}$ \\
\hline $\begin{array}{l}\text { Hospitalization } \\
\text { for heart failure } \\
(\mathrm{HF})(37) \text {. }\end{array}$ & $\begin{array}{l}\text { Hospital admission (lasting at least } 24 \text { hours) for deteriorating } \\
\text { symptoms or signs of HF, where there is a documented } \\
\text { diagnosis of HF and the patient receives initiation or } \\
\text { intensification of treatment for HF. Initiation or intensification } \\
\text { of treatment includes at least one of the following: increase in } \\
\text { oral diuretic dose or addition of another oral diuretic, } \\
\text { intravenous diuretic therapy, intravenous vasoactive therapy } \\
\text { (vasodilator, inotrope or vasopressor), mechanical circulatory } \\
\text { support (MCS) (including intra-aortic balloon pump, Impella, } \\
\text { extra-corporeal membrane oxygenation) or cardiac } \\
\text { transplantation. } \\
\text { HF during or after the assigned PCI procedure itself is defined } \\
\text { as prolongation of the planned admission by at least } 24 \text { hours } \\
\text { due to acute heart failure requiring initiation or intensification } \\
\text { of treatment as defined above. Prolongation of hospital } \\
\text { admission in patients who have prophylactic pre-PCI insertion } \\
\text { of a MCS should not be recorded as having a HF hospitalization } \\
\text { unless there are features of HF requiring initiation or } \\
\text { intensification of treatment as defined above. } \\
\text { Elective admission for implantation or revision of ICD/cardiac } \\
\text { resynchronization therapy (CRT) devices will not constitute a } \\
\text { HF hospitalization endpoint. }\end{array}$ \\
\hline Major Bleeding & $\begin{array}{l}\text { Major bleeding will be defined using the Bleeding Academic } \\
\text { Research Consortium (BARC) categories(38) below: } \\
\text { Type 3a } \\
\text { - Overt bleeding plus haemoglobin drop of } \geq 30 \text { to } \\
<50 \mathrm{~g} / \mathrm{L} \text { (provided haemoglobin drop is related to } \\
\text { bleed) } \\
\text { - Any transfusion with overt bleeding } \\
\text { Type 3b } \\
\text { - Overt bleeding plus haemoglobin drop } \geq 50 \mathrm{~g} / \mathrm{L} \\
\text { (provided haemoglobin drop is related to bleed) } \\
\text { - Cardiac tamponade } \\
\text { Bleeding requiring surgical intervention for control } \\
\text { (excluding dental/nasal/skin/haemorrhoid) } \\
\text { Bleeding requiring intravenous vasoactive drugs } \\
\text { Type 3c } \\
\text { - Intracranial haemorrhage (does not include } \\
\text { microbleeds or haemorrhagic transformation; does } \\
\text { include intraspinal) }\end{array}$ \\
\hline
\end{tabular}




\begin{tabular}{|c|c|}
\hline & $\begin{array}{l}\text { - Subcategories; confirmed by autopsy or imaging or } \\
\text { lumbar puncture } \\
\text { - Intra-ocular bleed compromising vision } \\
\text { Type 4: CABG-related bleeding } \\
\text { - Perioperative intracranial bleeding within } 48 \text { hours } \\
\text { - Reoperation following closure of sternotomy for the } \\
\text { purpose of controlling bleeding } \\
\text { - Transfusion of } \geq 5 \text { units of whole blood or packed red } \\
\text { blood cells within a } 48 \text { period } \\
\text { - Chest tube output } \geq 2 \text { L within a } 24 \text { h period } \\
\text { - If a CABG-related bleed is not adjudicated as at least a } \\
\text { Type } 3 \text { severity event, it will be classified as 'not a } \\
\text { bleeding event' } \\
\text { Type 5: fatal bleeding } \\
\text { Type 5a } \\
\text { - Probable fatal bleeding: no autopsy or imaging } \\
\text { confirmation, but clinically suspicious } \\
\text { Type 5b } \\
\text { - Definite fatal bleeding: overt bleeding or autopsy or } \\
\text { imaging confirmation }\end{array}$ \\
\hline $\begin{array}{l}\text { Unplanned } \\
\text { revascularisation }\end{array}$ & $\begin{array}{l}\text { PCI group: any unplanned target vessel or non-target vessel } \\
\text { revascularisation by PCI or CABG following index PCI, } \\
\text { excluding provisional staged PCI (with plan documented at the } \\
\text { index procedure). } \\
\text { OMT group: any revascularisation by PCI or CABG }\end{array}$ \\
\hline
\end{tabular}

